\title{
Measurement of serum PODXL concentration for detection of pancreatic cancer
}

This article was published in the following Dove Press journal:

OncoTargets and Therapy

\author{
Keisuke Taniuchi ${ }^{1,2}$ \\ Makiko Tsuboi \\ Masahiko Sakaguchi ${ }^{3,4}$ \\ Toshiji Saibara ${ }^{1,2}$ \\ 'Department of Endoscopic \\ Diagnostics and Therapeutics, Kochi \\ Medical School, Kochi University, \\ Nankoku, Kochi, Japan; ${ }^{2}$ Department \\ of Gastroenterology and Hepatology, \\ Kochi Medical School, Kochi \\ University, Nankoku, Kochi, Japan; \\ ${ }^{3}$ Integrated Center for Advanced \\ Medical Technologies, Kochi Medical \\ School, Kochi University, Nankoku, \\ Kochi, Japan; ${ }^{4}$ Cancer Prevention and \\ Control Division, Kanagawa Cancer \\ Center Research Institute, Yokohama, \\ Kanagawa, Japan
}

Background: The aim of this study was to investigate the use of podocalyxin (PODXL) and secretoglobin family 1D, member 2 (SCGB1D2) expressions in whole blood as diagnostic biomarkers to distinguish between patients with pancreatic cancer and control participants, in comparison with serum cancer antigen 19-9 (CA19-9), which is the current clinical standard.

Patients and methods: Flow cytometric analysis was performed to determine the expressions of PODXL and SCGB1D2 on the surface of cultured pancreatic cancer cells. Immunoblotting was performed to determine whether PODXL and SCGB1D2 were detectable in the media of cultured pancreatic cancer cells. A discovery-stage clinical study was performed in a cohort of 23 patients with pancreatic cancer and 51 control individuals without pancreatic disease who had been treated in the Department of Gastroenterology and Hepatology at Kochi Medical School Hospital from April 2014 to January 2016. Serum PODXL and SCGB1D2 levels were measured by enzyme-linked immunosorbent assay (ELISA).

Results: PODXL and SCGB1D2 accumulated in the protrusions of cultured pancreatic cancer cells, and they were detectable both on the cell surface and in the cultured media from these cells. The discovery-stage clinical study showed that the area under the receiver-operating characteristic curve (AUC) was 0.96 (95\% confidence interval [CI] 0.91-1.000) for PODXL, 0.80 (95\% CI 0.67-0.94) for SCGB1D2, and 0.78 (95\% CI 0.66-0.90) for CA19-9. The AUC for PODXL was thus significantly higher than that for CA19-9 $(P=0.006)$. The combination of SCGB1D2 with CA19-9 did not significantly increase the AUC $(0.83$; 95\% CI 0.70-0.96) compared with the AUC for either SCGB1D2 or CA19-9 alone $(P=0.563)$.

Conclusion: PODXL may be a novel, non-invasive diagnostic biomarker for the detection of pancreatic cancer.

Keywords: podocalyxin-like protein, secretoglobin, pancreatic cancer, diagnostic marker

\section{Introduction}

Pancreatic ductal adenocarcinoma (PDAC) is the fourth most common cause of cancer death in the Western world. ${ }^{1}$ The prognosis is poor, with 1 - and 5-year survival rates of only $20 \%$ and $6 \%$, respectively. ${ }^{2}$ Early diagnosis of PDAC is difficult, and there are no blood biomarkers that can assist in the identification of such patients at an early stage. ${ }^{3}$ Therefore, the discovery of blood biomarkers that can improve the diagnosis of PDAC and provide prognostic information for these individuals would greatly assist patient management.

We have previously described two glycoproteins, podocalyxin (PODXL) and secretoglobin family 1D, member 2 (SCGB1D2), which promote the motility and invasiveness of PDAC cells. ${ }^{4,5}$ PODXL is a highly glycosylated and sialylated transmembrane protein. ${ }^{6}$ High PODXL expression is associated with a significantly higher risk of death from PDAC, ${ }^{4,7}$ as well as poor differentiation, perineural invasion, and
Correspondence: Keisuke Taniuchi Department of Endoscopic Diagnostics and Therapeutics, Kochi Medical School, Kochi University, Kohasu, Oko-cho, Nankoku, Kochi 783-8505, Japan Tel/fax +8I 888802338

Email ktaniuchi@kochi-u.ac.jp (c)
hereby accept the Terms. Non-commercial uses of the work are permitted without any further permission from Dove Medical Press Limited, provided the work is properly attributed. For permission hereby accept the Terms. Non-commercial uses of the work are permitted without any further permission from Dove Medicat 
perivascular invasion. ${ }^{7}$ PODXL contributes to the formation of additional membrane protrusions in PDAC cells through the recruitment of gelsolin to filamentous actin, resulting in increased motility and invasiveness. ${ }^{4}$ PODXL is thus an important marker of poor prognosis in PDAC. ${ }^{4}$

SCGB1D2, also termed lipophilin B, is a member of the secretoglobin (SCGB) superfamily, a group of small, secretory, rarely glycosylated, dimeric proteins with unclear physiological functions, mainly expressed in mucosal tissues. ${ }^{8}$ Mature SCGBs are secreted proteins composed of homo- and heterodimers of SCGB polypeptides. ${ }^{9}$ SCGB1D2 is expressed in a high proportion of breast cancers, ${ }^{10}$ and it is more frequently expressed in estrogen receptor-positive tumors. ${ }^{11}$ We recently reported that, like PODXL, SCGB1D2 is also a biomarker of poor prognosis in PDAC and plays a role in the formation of cell protrusions important for cancer cell migration. ${ }^{5}$

Although it was discovered $>30$ years ago, serum cancer antigen 19-9 (CA19-9) remains the gold standard serum marker for patients with PDAC. In a cohort where approximately half of the patients had Stage IV disease, CA19-9 was found to have a median sensitivity of 79\% (70\%-90\%) and a median specificity of $82 \%(68 \%-91 \%)$ for PDAC diagnosis; however, the marker is not useful in the mass screening of asymptomatic subjects. ${ }^{12}$ Moreover, inadequate sensitivity and specificity limit the use of CA19-9 in the early diagnosis of PDAC. ${ }^{13}$ Several other potential serum or tissue markers for PDAC, such as TIMP-1 and ICAM-1, are currently being evaluated. ${ }^{14}$ Overall, however, in current clinical practice, biomarkers have a limited role to play in diagnosing PDAC.

The aim of the present study was to assess the utility of serum PODXL and SCGB1D2 as diagnostic markers for differentiating PDAC from control participants without pancreatic disease. The differential expressions of PODXL and SCGB1D2 in serologic samples of patients with earlystage (0, I, and II) and late-stage (III and IV) PDAC are also reported.

\section{Patients and methods Cell culture}

The human PDAC cell line S2-013, a subline of SUIT-2, was obtained from Dr T Iwamura (Miyazaki Medical College, Miyazaki, Japan). ${ }^{15}$ The human PDAC cell line, PANC-1, was purchased from the American Type Culture Collection (Manassas, VA, USA). HPNE immortalized normal pancreatic epithelial cells were a kind gift from Dr Ouellette. ${ }^{16}$ The ethics review board of Kochi Medical School approved the use of the gifted cell lines. All cells were grown in Dulbecco's Modified Eagle's Medium (DMEM; Thermo Fisher Scientific, Waltham, MA, USA) supplemented with $10 \%$ heat-inactivated fetal calf serum at $37^{\circ} \mathrm{C}$ in a humidified atmosphere saturated with $5 \% \mathrm{CO}_{2}$.

\section{Confocal immunofluorescence microscopy}

Immunocytochemistry was carried out, as previously described. ${ }^{17}$ Briefly, coverslips were coated with $10 \mu \mathrm{g} / \mathrm{mL}$ of fibronectin (Sigma-Aldrich Co., St Louis, MO, USA) for $1 \mathrm{~h}$ at room temperature. Cells were seeded on fibronectincoated glass coverslips, incubated for $5 \mathrm{~h}$, and then fixed with $4 \%$ paraformaldehyde and permeabilized with $0.1 \%$ Triton X-100. Blocking solution (3\% bovine serum albumin/ PBS) was added, followed by incubation with anti-PODXL antibody (PAB20111; Abnova, Taipei, Taiwan) or antiSCGB1D2 antibody (sc-48327; Santa Cruz Biotechnology Inc., Dallas, TX, USA) for $1 \mathrm{~h}$. After washing, Alexa488and Alexa594-conjugated secondary antibody (Molecular Probes, Carlsbad, CA, USA) was applied. Each specimen was visualized using a Zeiss LSM 510 META microscope (Carl Zeiss Meditec AG, Jena, Germany).

\section{Flow cytometric analysis}

S2-013 and HPNE cells $\left(1 \times 10^{6}\right.$ cells $)$ were incubated with anti-PODXL antibody (NBP2-27219; Novus Biologicals, Littleton, CO, USA) or anti-SCGB1D2 antibody (sc-48327) at $4^{\circ} \mathrm{C}$ for $1 \mathrm{~h}(0.34 \mathrm{mg} / \mathrm{mL})$. Mouse IgM for PODXL and goat IgG for SCGB1D2 were used as isotype controls ( $0.34 \mathrm{mg} / \mathrm{mL}$, Santa Cruz Biotechnology Inc.). The cells were washed in PBS and then incubated with Alexa488-conjugated secondary antibodies (Molecular Probes) at $4^{\circ} \mathrm{C}$ for $30 \mathrm{~min}$. The cells were washed in PBS and analyzed using a FACScan flow cytometer (BD Biosciences, San Jose, CA, USA) and ModFit (Verity Software House Inc., Topsham, ME, USA). Mean fluorescence intensity was calculated as relative signal intensity, ie, cells treated with anti-PODXL/cells treated with mouse IgM isotype control or cells treated with antiSCGB1D2/cells treated with goat IgG isotype control.

\section{Immunoblot analysis of cell lysates}

Cell pellets were resuspended in $20 \mathrm{mM}$ HEPES ( $\mathrm{pH} 7.4$ ), $100 \mathrm{mM} \mathrm{KCl}, 2 \mathrm{mM} \mathrm{MgCl}_{2}, 0.5 \%$ Triton X-100, protease inhibitor cocktail tablets (Hoffman-La Roche Ltd., Basel, Switzerland), and a phosphatase inhibitor cocktail (Nacalai Tesque, Kyoto, Japan). The bicinchoninic acid (BCA) assay was used to determine the protein concentration of each lysate; 
an aliquot of each lysate was then diluted with sample buffer (50 $\mathrm{mM}$ Tris, $2 \%$ sodium dodecyl sulfate [SDS], $0.1 \%$ bromophenol blue, and $10 \%$ glycerol) to a final concentration of $1-2 \mu \mathrm{g} / \mu \mathrm{L}$, and it was then analyzed by SDS-polyacrylamide gel electrophoresis (PAGE) and Western blotting using anti-PODXL (PAB20111), anti-SCGB1D2 (sc-48327), and anti-GAPDH (014-25524; Wako Pure Chemical Industries, Ltd., Osaka, Japan) antibodies.

\section{Immunoblot analysis of culture conditioned media}

S2-013 and HPNE cells were grown to $70 \%$ confluency, washed three times with PBS, and incubated for $48 \mathrm{~h}$ in the presence of conditioned media supplemented with $1 \%$ heatinactivated fetal calf serum. Culture-conditioned media were collected, and cell cultures were trypsinized and analyzed for the presence of necrotic cells using trypan blue staining. Culture-conditioned media harvested from S2-013 and HPNE cells were centrifuged using a Beckman Coulter Allegra $\mathrm{X}-15 \mathrm{R}$ centrifuge at $300 \times \mathrm{g}$ at $4^{\circ} \mathrm{C}$ for $10 \mathrm{~min}$ to remove detached cells. Supernatant was collected and filtered through $0.22 \mu \mathrm{m}$ filters (Merck Millipore, Billerica, MA, USA) to remove contaminating apoptotic bodies, microvesicles, and cell debris. Next, the samples were concentrated using an Amicon Ultra 10K Centrifugal Filter (Merck Millipore), according to the manufacturer's instructions. The concentrations of PODXL and SCGB1D2 in these concentrated conditioned media were determined by immunoblotting. An aliquot of each conditioned medium was diluted with sample buffer (50 mM Tris, 2\% SDS, 0.1\% bromophenol blue, and 10\% glycerol) and analyzed by SDS-PAGE and Western blotting using anti-PODXL (PAB20111), anti-SCGB1D2 (sc-48327), anti-GAPDH (014-25524), and anti- $\alpha$-tubulin (017-25031; Wako Pure Chemical Industries, Ltd.) antibodies.

\section{Human serum PDAC samples for enzyme-linked immunosorbent assay (ELISA)}

The serum PODXL and SCGB1D2 levels and the clinicopathological data were analyzed retrospectively. All serum samples analyzed by ELISA were collected in the Kochi Medical School Hospital from April 2014 to January 2016. Serum samples from PDAC patients were selected for the study on the basis of the following criteria: 1) patients who were newly diagnosed and previously untreated, and 2) those with tumors pathologically diagnosed as PDAC. Tumors were classified (Stages I-IV) according to the classification of the International Union against Cancer (Table 1). ${ }^{18}$
Table I Summary of characteristics

\begin{tabular}{|c|c|c|}
\hline Characteristics & PDAC, \% (n) & Control, \% (n) \\
\hline \multicolumn{3}{|l|}{ Age, years } \\
\hline$\leq 39$ & $0(0)$ & $7.8(4)$ \\
\hline $40-49$ & $4.3(I)$ & II.7 (6) \\
\hline $50-59$ & $4.3(I)$ & $9.8(5)$ \\
\hline $60-69$ & $26.0(6)$ & $4 I .2(2 I)$ \\
\hline 70-79 & $56.5(13)$ & $13.7(7)$ \\
\hline$\geq 80$ & $8.7(2)$ & $15.7(8)$ \\
\hline \multicolumn{3}{|l|}{ Gender } \\
\hline Male & $52.2(12)$ & $43.1(22)$ \\
\hline Female & $47.8(\mathrm{II})$ & $56.9(29)$ \\
\hline \multicolumn{3}{|l|}{ Diagnosed with diabetes } \\
\hline Yes & $21.7(5)$ & I3.7 (7) \\
\hline No & $78.3(18)$ & $86.3(44)$ \\
\hline \multicolumn{3}{|l|}{ Diagnosed with hypertension } \\
\hline Yes & $43.5(10)$ & $37.3(19)$ \\
\hline No & $56.5(13)$ & $62.7(32)$ \\
\hline \multicolumn{3}{|l|}{ Serum uric acid } \\
\hline Upregulated & $13.0(3)$ & $17.6(9)$ \\
\hline Normal range & $87.0(20)$ & $82.4(42)$ \\
\hline \multicolumn{3}{|l|}{ Serum triglyceride } \\
\hline Upregulated & $8.7(2)$ & $31.4(16)$ \\
\hline Normal range & $91.3(2 \mathrm{I})$ & $68.6(35)$ \\
\hline \multicolumn{3}{|l|}{ Stage } \\
\hline 0 & $4.3(I)$ & \\
\hline IA & $0(0)$ & \\
\hline IB & $0(0)$ & \\
\hline$\| \mathrm{A}$ & $8.7(2)$ & \\
\hline IIB & $17.4(4)$ & \\
\hline III & $26.1(6)$ & \\
\hline IV & $43.5(10)$ & \\
\hline Serum CAI9-9, median (IQR) & $75.6(13.4-306.5)$ & I2.7 (0.93-28.4) \\
\hline
\end{tabular}

Note: 'Classified according to the classification of the International Union against Cancer. Abbreviations: CA19-9, cancer antigen 19-9; IQR, interquartile range; PDAC, pancreatic ductal adenocarcinoma.

Clinicopathological parameters were classified according to the pancreatic carcinoma criteria of the Japan Pancreas Society. ${ }^{19}$ All individuals classified as controls were diagnosed with benign gastrointestinal diseases such as gastric and colonic polyps while being evaluated for non-pancreatic diseases. The study was approved by the ethics review board of Kochi Medical School (approval number: ERB-101891), and the methods were carried out in accordance with the approved guidelines. Written informed consent was obtained from each patient. All serum samples were obtained at the time of diagnosis and stored at $-80^{\circ} \mathrm{C}$ until use.

\section{ELISA}

Serum levels of PODXL and SCGB1D2 were measured by ELISA using commercially available enzyme test kits (SEA768Hu [CLOUD-CLONE, Katy, TX, USA] for PODXL and CSB-EL020814HU [CUSABIO, Hubei Province, China] for SCGB1D2). All assays used a 
quantitative sandwich enzyme immunoassay technique. Serum samples were diluted 1 in 10 in assay buffer, and then $100 \mu \mathrm{L}$ of the diluted sample was incubated in precoated ELISA 96-well plates, along with standards, for $2 \mathrm{~h}$ at $37^{\circ} \mathrm{C}$. After washing the wells, $100 \mu \mathrm{L}$ of biotin-labeled polyclonal secondary antibody (detection reagent $\mathrm{A}$ ) was added and incubated for $1 \mathrm{~h}$ at $37^{\circ} \mathrm{C}$. After washing, $100 \mu \mathrm{L}$ of avidin-conjugated horseradish peroxidase (detection reagent $\mathrm{B}$ ) was added and incubated for $30 \mathrm{~min}$ at $37^{\circ} \mathrm{C}$. After a final washing step, $90 \mu \mathrm{L}$ of tetramethylbenzidine (TMB) substrate was added to each well and incubated for $\sim 15 \mathrm{~min}$ in the dark at $37^{\circ} \mathrm{C}$, until the second lowest standard could be distinguished from the blank by a change in color. Stopping solution ( $50 \mu \mathrm{L}$ of sulfuric acid) was then added, and the absorbance at $490 \mathrm{~nm}$ was analyzed using the SpectraMax 190 Microplate Reader (Molecular Devices LLC, Sunnyvale, CA, USA). Levels of CA19-9 in serum were measured by ELISA using a commercially available enzyme test kit (TM-CA-19-9 ELISA KIT; DRG International Inc., Springfield, NJ, USA), following the same protocol as mentioned earlier.

\section{Statistical analysis}

For the in vitro experiments, StarFlex software (version 6; YUMIT, Osaka, Japan) and SAS software (version 9.1.3; SAS Institute Inc., Cary, NC, USA) were used for statistical analysis. Student's $t$-test was used for the comparison of continuous variables. $P$-values $<0.05$ were considered significant, and all tests were two-tailed.

In the discovery stage clinical study, statistical analysis was performed using R (version 3.3.3; The R Foundation, Vienna, Austria). The pROC package (The R Foundation) was used for receiver operating characteristic (ROC) curve analysis. Patient sample characteristics, including PODXL, SCGB1D2, and CA19-9 expressions, were compared using the Wilcoxon rank sum test. Pearson's correlation coefficients were calculated for PODXL, SCGB1D2, and CA19-9. The predictive performances of PODXL and SCGB1D2 were evaluated using ROC analysis, the area under the receiver-operating characteristic curve (AUC), and the corresponding 95\% confidence intervals (CIs). DeLong's test was used for differences in AUC values. ${ }^{20}$ Multivariate logistic regression was used to establish the diagnostic mathematical model. The variance inflation factor (VIF) was used to check for multicollinearity using the DAAG package (The R Foundation). Diagnostic values of PODXL and SCGB1D2 were evaluated based on AUCs, with sensitivity and specificity evaluated at various cutoff values. The three cutoff values were calculated as: 1) the highest
Youden index, ${ }^{21}$ 2) the highest sensitivity with specificity not $<80 \%$, and 3 ) the highest specificity with sensitivity not $<80 \%$. The logit model was used to determine cutoff values for combinations of multiple biomarkers. ${ }^{22}$ In all analyses, $P$-values $<0.05$ were considered significant.

\section{Results \\ Extracellular localization of PODXL and SCGBID2 from cultured PDAC cells}

Immunocytochemistry was used to determine the subcellular localization of PODXL and SCGB1D2 in the moderately differentiated PDAC cell line (S2-013), the poorly differentiated PDAC cell line (PANC-1), and the immortalized normal pancreatic epithelial cell line (HPNE). Western blotting showed that S2-013 and PANC-1 cells expressed higher levels of PODXL and SCGB1D2 than HPNE cells (Figure 1A). Confocal microscopy showed that PODXL and SCGB1D2 were present in the cytoplasm of the cell bodies and in membrane protrusions in S2-013 and PANC-1 cells, which had many peripheral actin structures (Figure 1B). In contrast, there was no obvious staining of PODXL and SCGB1D2 in HPNE cells (Figure 1B).

The cell surface expressions of PODXL and SCGB1D2 were assessed in S2-013 and HPNE cells by flow cytometry. This demonstrated that PODXL and SCGB1D2 antibodies bound to the surface of S2-013 cells and that PODXL and SCGB1D2 were expressed on the surface of S2-013 cells (Figure 1C). PODXL and SCGB1D2 were not detectable in HPNE cells. Because PODXL is a transmembrane glycoprotein whose extracellular domain is modified heavily by $O$-linked glycosylation and the addition of highly charged sialic acid residues, ${ }^{23}$ and the extracellular domain of SCGB1D2 is thought to be secreted, ${ }^{8}$ immunoblotting was next used to examine the presence of these proteins in the culture media of S2-013 cells. The anti-PODXL antibody (PAB20111) recognized the extracellular domain of PODXL. Western blotting showed that PODXL and SCGB1D2 were both detected in media from S2-013 cultures (Figure 1D). In contrast, PODXL and SCGB1D2 were not detectable in media from HPNE cells (Figure 1D). We used two loading control markers, glyceraldehyde-3-phosphate dehydrogenase (GAPDH) and $\alpha$-tubulin. GAPDH with the same molecular mass as the intracellular GAPDH is detected in the conditioned medium of mammalian cultured cell lines, ${ }^{24}$ and $\alpha$-tubulin is an intracellular protein marker. GAPDH was abundantly present in media from S2-013 cultures, and $\alpha$-tubulin was not detectable in media from S2-013 and HPNE cultures (Figure 1D). 


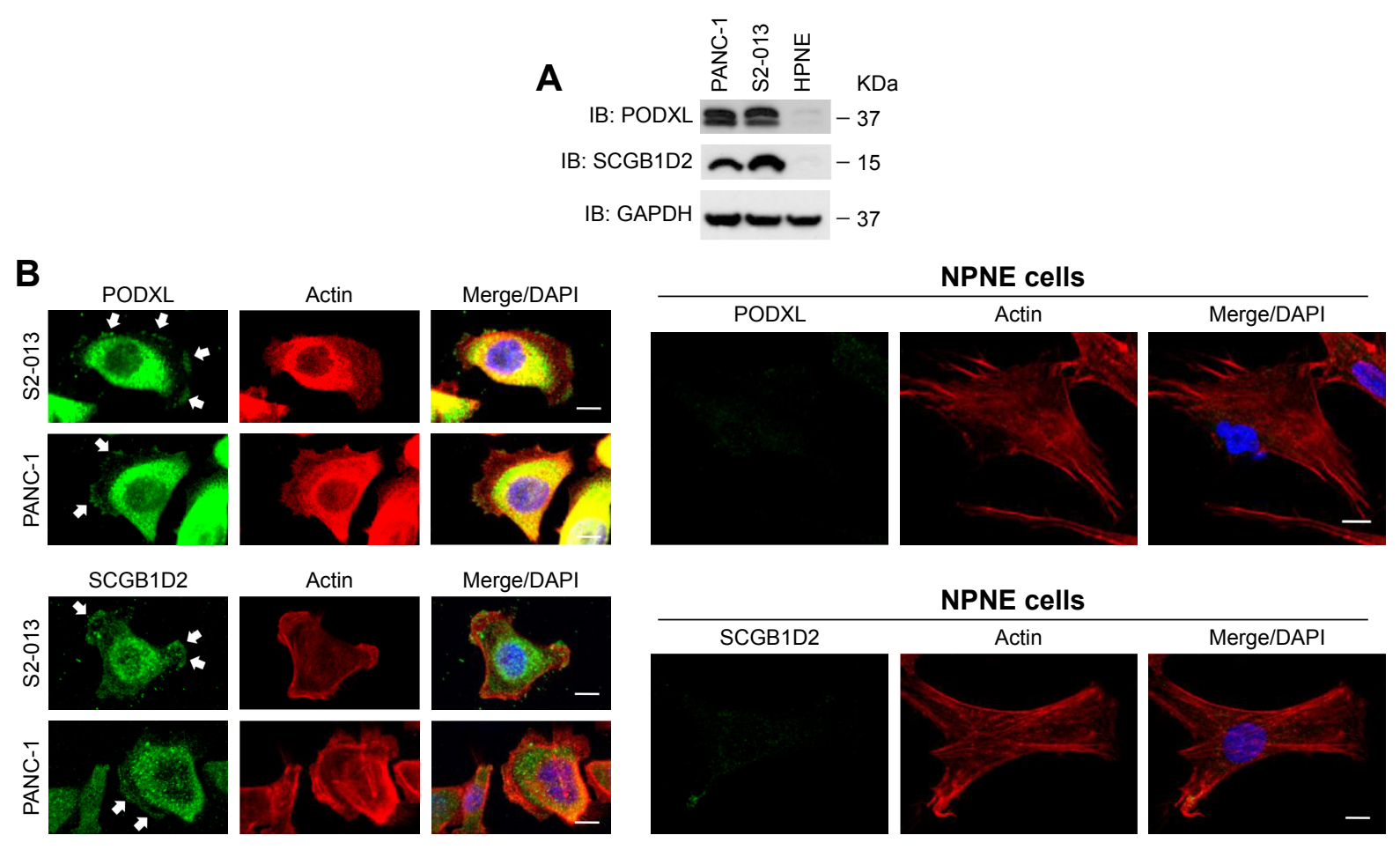

C
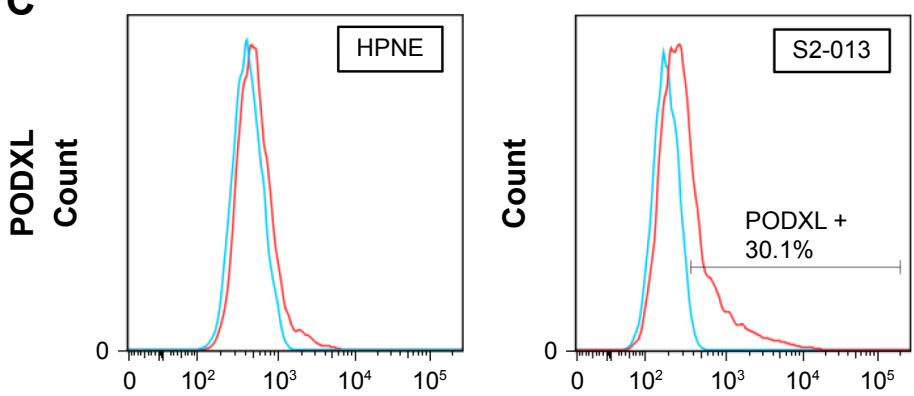

D
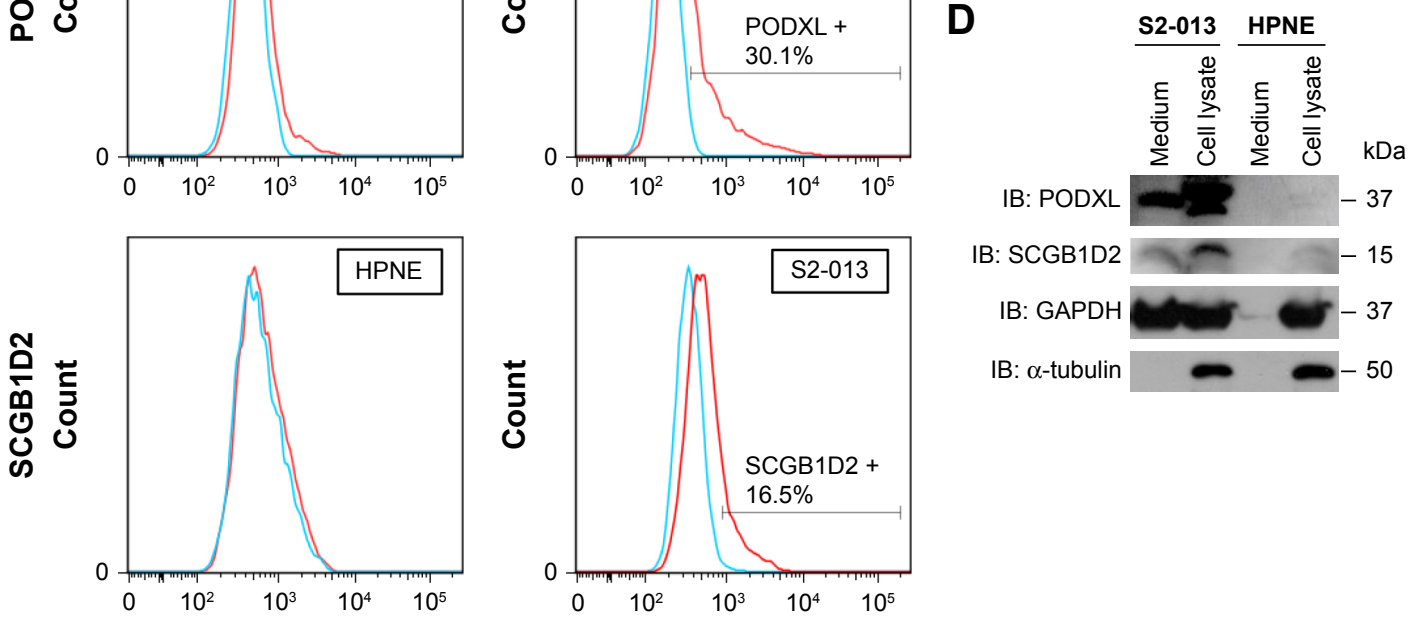

IB: PODXL

IB: SCGB1D2

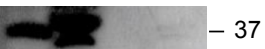

IB: GAPDH

IB: $\alpha$-tubulin

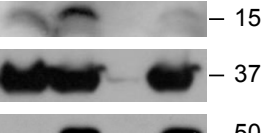

Figure I Subcellular localization of PODXL and SCGBID2 in PDAC and immortalized normal pancreatic epithelial cells.

Notes: (A) Western blotting of total cell lysates of S2-013, PANC-I, and HPNE cells was performed using anti-PODXL and anti-SCGBID2 antibodies. Loading control: GAPDH. (B) Confocal immunofluorescence microscopic images of S2-013, PANC-I, and HPNE cells labeled with anti-PODXL antibody (green) or anti-SCGBID2 (green), and phalloidin (red; actin filaments); arrows, PODXL and SCGBID2 localized to cell protrusions; blue, DAPI (nuclear) staining; bars, 10 $\mu \mathrm{m}$. (C) Expressions of PODXL and SCGBID2 on the surface of S2-013 and HPNE cells, as evaluated by flow cytometry. Cells treated with anti-PODXL or anti-SCGBID2 antibody (red) vs cells treated with the corresponding isotype control (blue). (D) Western blotting of total cell lysates and culture media of S2-0I3 and HPNE cells was performed using anti-PODXL and antiSCGBID2 antibodies. Loading control: $\alpha$-tubulin (an intracellular protein marker) and GAPDH.

Abbreviations: GAPDH, glyceraldehyde-3-phosphate dehydrogenase; PDAC, pancreatic ductal adenocarcinoma; PODXL, podocalyxin; SCGBID2, secretoglobin family ID, member 2.

\section{Serum levels of PODXL}

Because the in vitro findings suggested the potential use of PODXL as a novel tumor maker for PDAC, whether PODXL is present in the serum of patients with PDAC was investigated. The discovery-stage clinical study included a total of 74 serologic samples from patients with PDAC and control individuals without pancreatic disease. For individuals with no prior therapy for PDAC, 
disease staging (0-IV) for PDAC was performed at the time of specimen procurement. Clinical characteristics at the time of specimen procurement are summarized in Table 1. Of the individuals with PDAC, seven (30.4\%) had early-stage disease (0/I/II) at the time of specimen procurement.

The PODXL levels in serum samples from both PDAC patients and control individuals were detected by ELISA experiments; the median serum level of PODXL in PDAC patients was $8.74 \mathrm{ng} / \mathrm{mL}$ (interquartile range [IQR] 7.1-12.4), while in control individuals it was significantly lower (0.15 ng/mL; IQR -1.01 to 0.86 ) (Figure $2 \mathrm{~A}, P<0.001$ ). The detection sensitivity of the PODXL ELISA kit was $0.156 \mathrm{ng} / \mathrm{mL}$. The relationships between serum PODXL concentration and clinicopathological features were analyzed by the Wilcoxon rank sum test (Table 2). However, there were no significant associations between serum PODXL concentration and the clinical characteristics such as age, gender, tumor size, clinical stage, and serum CA19-9 levels in PDAC patients.

A

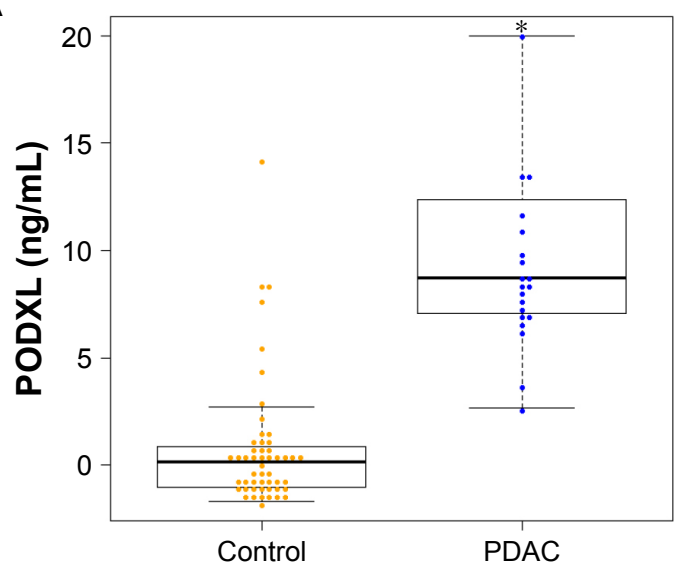

B

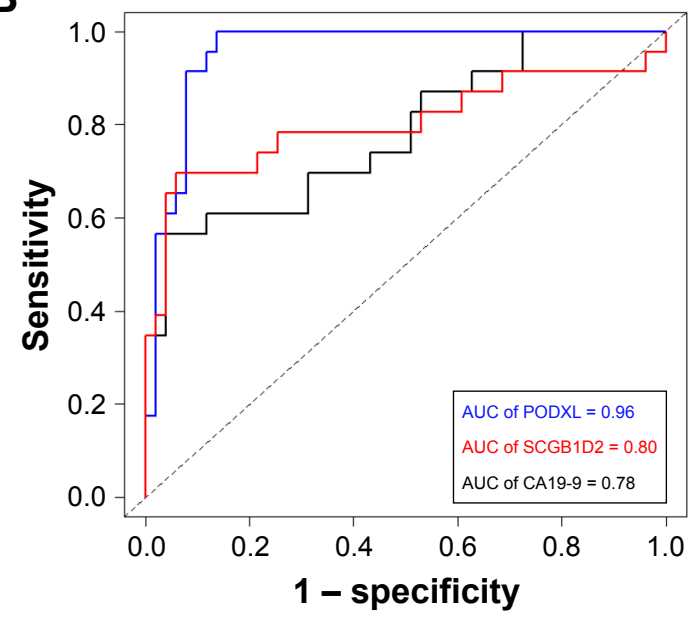

\section{Serum levels of SCGBID2}

The SCGB1D2 levels in serum samples of both PDAC patients and control individuals were detected by ELISA experiments; the median serum level of SCGB1D2 in PDAC patients was $238.87 \mathrm{ng} / \mathrm{mL}$ (IQR 115.11-683.74), while in control individuals it was $71.09 \mathrm{ng} / \mathrm{mL}$ (IQR 13.08-104.51) (Figure 2A, $P<0.001$ ). The relationship between SCGB1D2 levels and clinicopathological features is shown in Table 3. Serum SCGB1D2 level was significantly correlated with gender and serum triglyceride $(P<0.05)$. However, there were no significant associations between serum SCGB1D2 levels and other clinicopathological features such as tumor size, clinical stage, and serum CA19-9 levels in PDAC patients.

\section{ROC curve analyses}

ROC curves were used to evaluate the performance of PODXL and SCGB1D2 in distinguishing patients with PDAC from control individuals (Figure 2B). For the detection of PODXL and SCGB1D2 in PDAC and control individuals

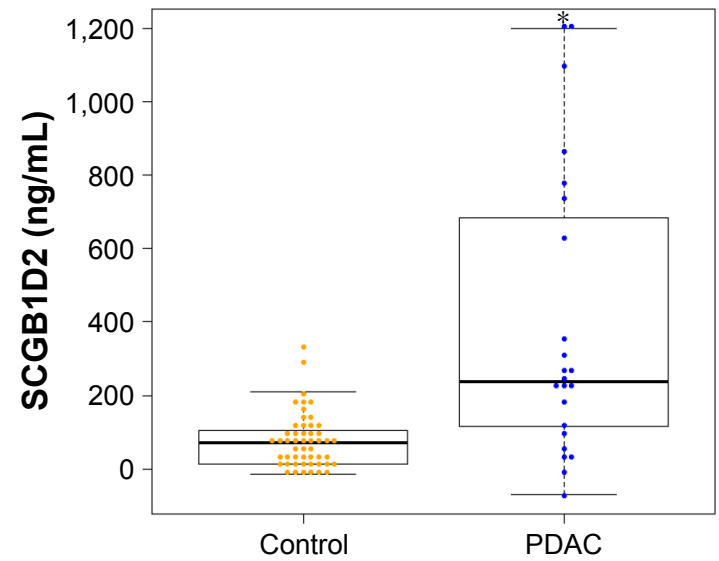

D

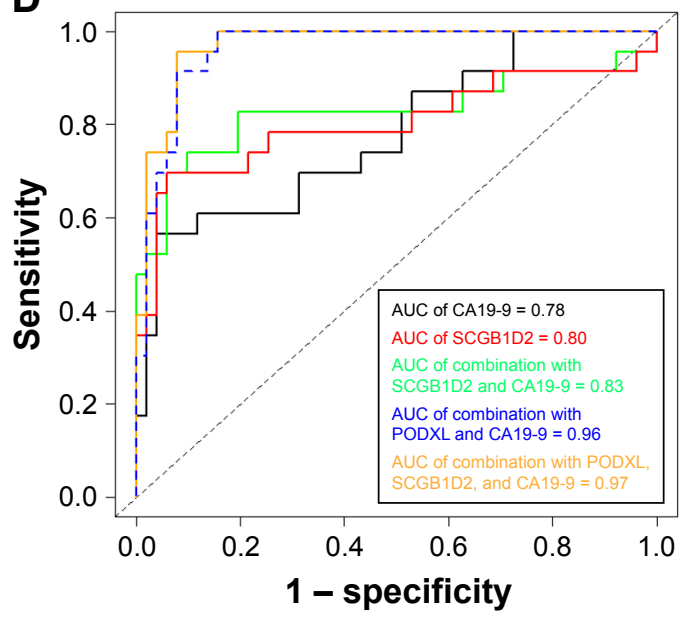

Figure 2 (Continued) 

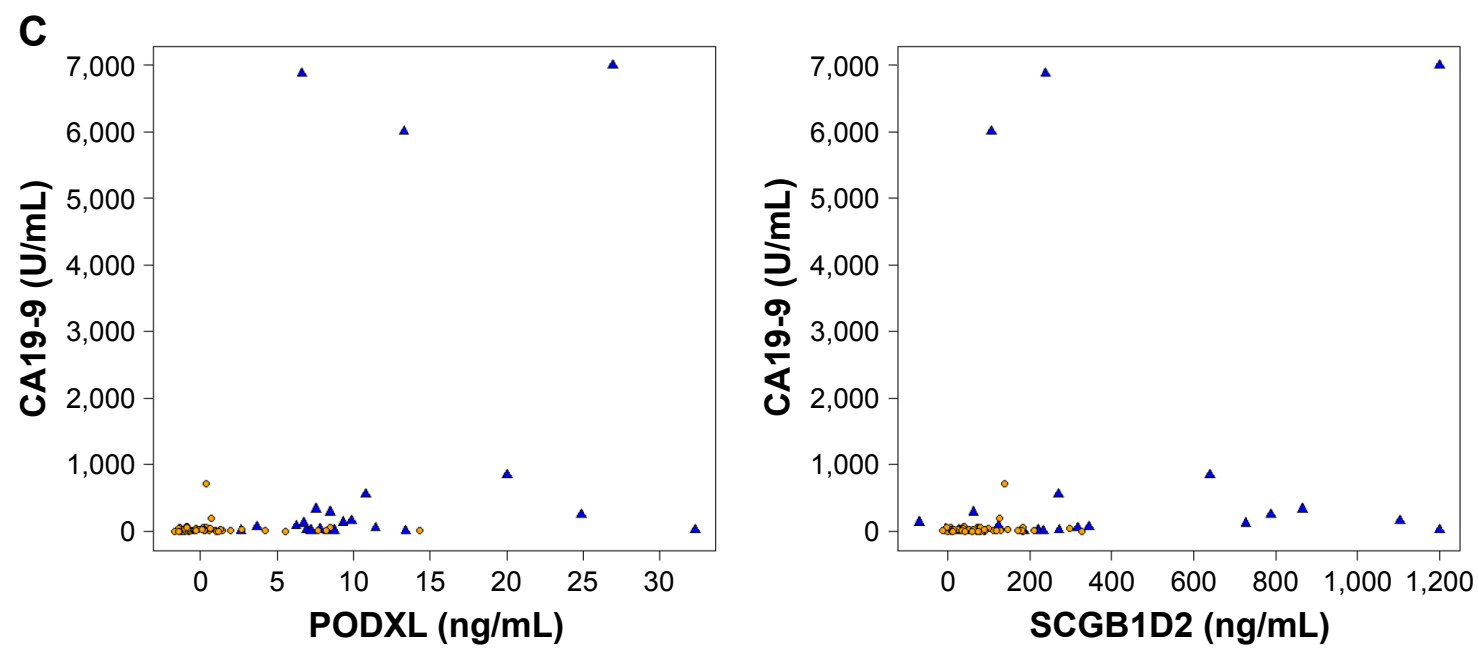

- Control $\triangle$ PDAC

Figure 2 The performance of PODXL, SCGBID2, CA19-9, and the combination of SCGBID2 with CA19-9 determined by ELISA for distinguishing PDAC patients from control individuals.

Notes: (A) Distribution of PODXL and SCGBID2 in sera from PDAC patients and control individuals. The horizontal line in the middle of each box indicates the median, whereas the top and bottom borders of the box mark the 75 th and 25 th percentiles, respectively. The upper whisker is the 75 th percentile $+($ I. $5 \times$ IQR). The lower whisker is the 25th percentile $-(I .5 \times I Q R)$. Differences are significant between PDAC patients and control individuals $(* P=0.00 I$, Mann-Whitney $U$ test). (B) ROC curves of the levels of PODXL, SCGBID2, and CAI9-9 in sera from PDAC patients and control individuals; $X$-axis, I - specificity; $Y$-axis, sensitivity. ( $* P=0.001$, Mann-Whitney $U$ test) (C) Relationships between serum levels of PODXL, SCGBID2, and CAI9-9 (X-axis, CAI9-9 concentration; $Y$-axis, PODXL or SCGBID2 concentration). (D) ROC curves for the performance of SCGBID2 and CA19-9 alone, the combination of SCGBID2 with CAI9-9, the combination of PODXL with CAI9-9, and the combination of PODXL and SCGBID2 with CAI9-9.

Abbreviations: CAI9-9, cancer antigen 19-9; ELISA, enzyme-linked immunosorbent assay; GAPDH, glyceraldehyde-3-phosphate dehydrogenase; IQR, interquartile range; PDAC, pancreatic ductal adenocarcinoma; PODXL, podocalyxin; ROC, receiver operating characteristic; SCGBID2, secretoglobin family ID, member 2.

Table 2 Correlation between serum PODXL level and clinicopathological parameters in PDAC

\begin{tabular}{|c|c|c|c|}
\hline \multirow[t]{2}{*}{ Characteristics } & \multicolumn{2}{|c|}{$\begin{array}{l}\text { Serum PODXL level } \\
(\mathrm{ng} / \mathrm{mL})\end{array}$} & \multirow[t]{2}{*}{$P$-value } \\
\hline & Median & IQR & \\
\hline Age, years & & & 0.699 \\
\hline$<70(\mathrm{n}=8)$ & 8.6 & $7.4-10.3$ & \\
\hline$\geq 70(n=15)$ & 8.7 & $7.1-15.7$ & \\
\hline Gender & & & 0.310 \\
\hline Male $(n=12)$ & 9.3 & $7.4-15.0$ & \\
\hline Female $(n=I I)$ & 8.4 & $6.7-10.4$ & \\
\hline Diagnosed with diabetes & & & 0.766 \\
\hline Yes $(n=5)$ & 8.7 & $6.7-11.4$ & \\
\hline No $(n=18)$ & 8.6 & $7.3-12.7$ & \\
\hline Diagnosed with hypertension & & & 0.321 \\
\hline Yes $(n=10)$ & 7.7 & $6.8-9.6$ & \\
\hline No $(n=13)$ & 9.3 & $8.4-13.3$ & \\
\hline Serum uric acid & & & 0.553 \\
\hline Upregulated $(n=3)$ & 10.8 & $9.3-12.0$ & \\
\hline Normal range $(n=20)$ & 8.6 & $6.9-11.9$ & \\
\hline Serum triglyceride & & & 0.785 \\
\hline Upregulated $(n=2)$ & 8.3 & $7.7-8.8$ & \\
\hline Normal range $(n=2 \mathrm{I})$ & 8.7 & $6.9-13.3$ & \\
\hline Stage $^{\mathrm{a}}$ & & & 0.973 \\
\hline $0, I$, II (n=7) & 8.5 & $7.0-18.2$ & \\
\hline III, IV (n=I6) & 8.7 & $7.1-11.4$ & \\
\hline Tumor diameter & & & 0.137 \\
\hline $0-3 \mathrm{~cm}(\mathrm{n}=13)$ & 9.3 & $8.4-13.3$ & \\
\hline$>3 \mathrm{~cm}(\mathrm{n}=10)$ & 7.1 & $6.7-10.3$ & \\
\hline
\end{tabular}

Note: ${ }^{2}$ Classified according to the classification of the International Union against Cancer. Abbreviations: IQR, interquartile range; PDAC, pancreatic ductal adenocarcinoma; PODXL, podocalyxin.
Table 3 Correlation between serum SCGBID2 level and clinicopathological parameters in PDAC

\begin{tabular}{|c|c|c|c|}
\hline \multirow[t]{2}{*}{ Characteristics } & \multicolumn{2}{|c|}{$\begin{array}{l}\text { Serum SCGB I D2 } \\
\text { level (ng/mL) }\end{array}$} & \multirow[t]{2}{*}{$P$-value } \\
\hline & Median & IQR & \\
\hline Age, years & & & 0.438 \\
\hline$<70(\mathrm{n}=8)$ & 203.0 & $94.9-440.0$ & \\
\hline$\geq 70(n=15)$ & 269.7 & $|72.4-7| 4.3$ & \\
\hline Gender & & & 0.023 \\
\hline Male $(n=12)$ & 455.6 & 234.2-808.I & \\
\hline Female $(n=I I)$ & 124.5 & $33.5-274.6$ & \\
\hline Diagnosed with diabetes & & & 0.502 \\
\hline Yes $(n=5)$ & 317.1 & 238.9-344.I & \\
\hline No $(n=18)$ & 226.2 & $73.4-773.6$ & \\
\hline Diagnosed with hypertension & & & 0.457 \\
\hline Yes $(n=10)$ & 291.5 & $85.6-1,044.5$ & \\
\hline No $(n=13)$ & 232.1 & $|24.5-3| 7 . \mid$ & \\
\hline Serum uric acid & & & 0.185 \\
\hline Upregulated $(n=3)$ & 105.7 & $49.8-187.7$ & \\
\hline Normal range $(n=20)$ & 255.1 & | $70.3-743.0$ & \\
\hline Serum triglyceride & & & 0.043 \\
\hline Upregulated $(n=2)$ & -15.0 & $-42.8-12.8$ & \\
\hline Normal range $(n=2 I)$ & 269.7 & 185.6-727.7 & \\
\hline Stage $^{\mathrm{a}}$ & & & 0.504 \\
\hline $0, \mathrm{I}, \mathrm{II}(\mathrm{n}=7)$ & 124.5 & $44.5-553.0$ & \\
\hline III, IV $(n=16)$ & 254.3 & $211.7-661.7$ & \\
\hline Tumor diameter & & & 0.598 \\
\hline $0-3 \mathrm{~cm}(\mathrm{n}=13)$ & 220.3 & 105.7-788.9 & \\
\hline$>3 \mathrm{~cm}(\mathrm{n}=10)$ & 270.5 & $225.0-565.8$ & \\
\hline
\end{tabular}

Note: ${ }^{a}$ Classified according to the classification of the International Union against Cancer. Abbreviations: IQR, interquartile range; PDAC, pancreatic ductal adenocarcinoma; SCGBID2, secretoglobin family ID, member 2 . 
without pancreatic disorders, the AUC was 0.96 (95\% CI $0.91-0.99)$ and 0.80 (95\% CI $0.67-0.94)$, respectively. The levels of CA19-9 were determined using a commercial ELISA kit, and the AUC of CA19-9 was 0.78 (95\% CI 0.66-0.90). The performance of PODXL in distinguishing PDAC from control individuals was found to be superior to that of CA19-9 $(P=0.006)$. However, SCGB1D2 was not superior to CA19-9 $(P=0.802)$.

The sensitivity and specificity of PODXL for PDAC diagnosis were analyzed using different cutoff values (Table 4). Three cutoff values for each marker were obtained by minimizing the total prediction error, as described in the "Patients and methods" section. The sensitivity of PODXL ranged from $91.3 \%$ to $100 \%$, and the specificity varied from $86.3 \%$ to $92.2 \%$, for the different cutoff values. Specifically, when a threshold of $6.27 \mathrm{ng} / \mathrm{mL}$ was used, a sensitivity of $91.3 \%$, a specificity of $92.2 \%$, and an accuracy of $91.9 \%$ were achieved (Table 4). The sensitivity of SCGB1D2 ranged from $69.6 \%$ to $82.6 \%$, and the specificity varied from $47.1 \%$ to $94.1 \%$ for the different cutoff values. Specifically, when a threshold of $185.58 \mathrm{ng} / \mathrm{mL}$ was used, a sensitivity of $69.6 \%$, a specificity of $94.1 \%$, and an accuracy of $86.5 \%$ were achieved (Table 4). Using the same method for CA19-9, instead of using the standard cutoff value $(37.0 \mathrm{U} / \mathrm{mL})$, when a threshold of $66.0 \mathrm{U} / \mathrm{mL}$ was used, a sensitivity of $56.5 \%$, a specificity of $96.1 \%$, and an accuracy of $83.8 \%$ were achieved (Table 4). Decreasing the cutoff value to $50.0 \mathrm{U} / \mathrm{mL}$ improved the sensitivity for the detection of PDAC to $60.9 \%$, but lowered the specificity to $88.2 \%$ and the accuracy to $79.7 \%$.

\section{Combination with SCGBID2 and CAI9-9}

There were significant correlations between the serum levels of PODXL and CA19-9 (Pearson's correlation coefficient $[R]=0.38,95 \%$ CI $0.17-0.56, P=0.007)$, between SCGB1D2 and CA19-9 $(R=0.31,95 \%$ CI $0.09-0.50, P=0.007)$, and between PODXL and SCGB1D2 $(R=0.72,95 \% \mathrm{CI}$ $0.588-0.81, P<0.001$ ) (Figure $2 \mathrm{C}$ ). To take account of multicollinearity, VIF was employed. None of the VIF values for the two models reached a value of 10 , meaning that there was no collinearity in the model. VIF values for CA19-9, PODXL, gender, and age were 1.02, 1.08, 1.07, and 1.01, respectively. VIF values for CA19-9, SCGB1D2, gender, and age were $1.05,1.25,1.24$, and 1.04, respectively.

SCGB1D2 and CA19-9 were combined in an attempt to improve the sensitivity and specificity for the detection of PDAC. ROC analysis showed that the AUC for the

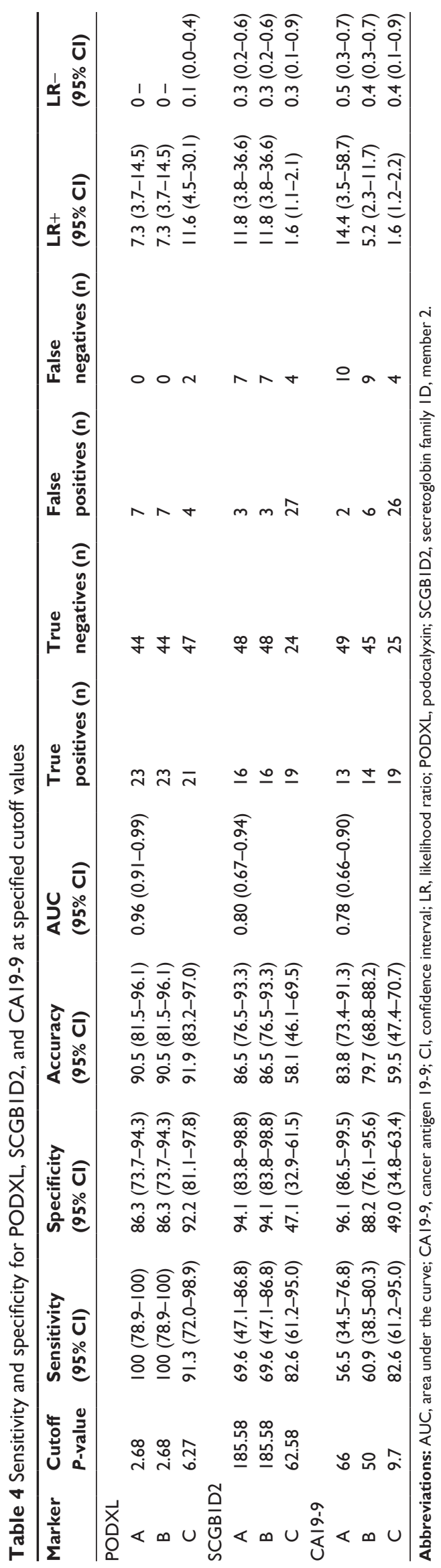


combination of SCGB1D2 and CA19-9 was 0.83 (95\% CI $0.70-0.96)$, which was not significantly different from the AUC of SCGB1D2 alone $(P=0.213)$ or CA19-9 alone $(P=0.563)$ (Figure 2D). The sensitivity of the combination of SCGB1D2 and CA19-9 ranged from $73.9 \%$ to $82.6 \%$, the specificity ranged from $80.4 \%$ to $90.2 \%$, and the accuracy ranged from $81.1 \%$ to $85.1 \%$ for the different cutoff values (Table 5). When a threshold of 0.306 was used, a sensitivity of $73.9 \%$, a specificity of $90.2 \%$, and an accuracy of $85.1 \%$ were achieved (Table 5). With the combination of PODXL and CA19-9, the AUC of this combination was not significantly different from the AUC of PODXL alone (Figure 2D), indicating that PODXL was independently able to distinguish PDAC patients from control individuals.

\section{Association of serum tumor marker levels with the risk of PDAC}

Next, the significance of serum tumor marker levels for the diagnosis of PDAC was evaluated using logistic regression to obtain crude odds ratios (ORs) (Table 6). To exclude the possible effects of age and gender, crude ORs were then adjusted for the effects of age and gender (adjusted OR [aOR] values). The results showed that among PODXL, SCGB1D2, and CA19-9, the serum level of PODXL was the most highly correlated with the risk of PDAC. The combination of PODXL with CA19-9 did not improve PDAC detection compared to the use of PODXL alone (aOR of CA19-9 $=1.00,95 \%$ CI $0.99-1.01, P=0.227$, for the multivariate regression model of PODXL and CA19-9).

\section{Detection of early-stage PDACs using PODXL, SCGBID2, or CAI9-9}

The level of PODXL in serum samples from patients in the early stages of PDAC (Stages 0, I, and II) was significantly higher than the level in controls, as well as the level of CA19-9 in these patients (Figure 3A). ROC curves for PODXL, SCGB1D2, and CA19-9 are shown in Figure 3B. The AUC values of PODXL used to distinguish patients with Stage 0/I/II and Stage III/IV PDAC from controls were 0.96 and 0.96 , respectively (Table 7). For SCGB1D2, the AUC values used to distinguish patients with Stage 0/I/II and Stage III/IV PDAC from controls were 0.66 and 0.87, respectively (Table 7). For CA19-9, the AUC values to distinguish patients with Stage 0/I/II and Stage III/IV PDAC from controls were 0.79 and 0.77 , respectively (Table 7). The performance of PODXL in distinguishing PDAC at Stage 0/I/II from control individuals was found to be superior to that of CA19-9 $(P=0.048)$.

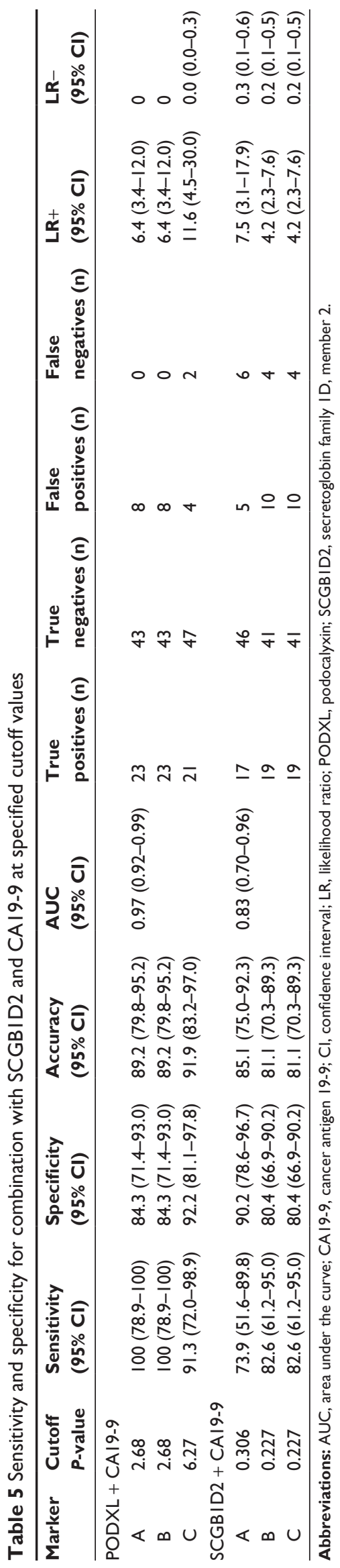


Table 6 Univariate and multivariate logistic regression analyses

\begin{tabular}{|c|c|c|c|c|c|c|}
\hline Indicators & Crude OR & $P$-value & Adjusted OR of Model I & $P$-value & Adjusted OR of Model 2 & $P$-value \\
\hline \multirow[t]{2}{*}{ PODXL } & 1.69 (95\% Cl I.35-2.12) & $<0.001$ & PODXL I.66 (95\% Cl I.32-2.I0) & $<0.001$ & PODXL I.67 (95\% Cl I.3I-2.13) & $<0.001$ \\
\hline & & & CAI9-9 I.00 (95\% Cl 0.99-I.0I) & 0.227 & CAI9-9 I.00 (95\% Cl 0.98-I.0I) & 0.225 \\
\hline \multirow[t]{2}{*}{ SCGBID2 } & I.0I (95\% Cl I.00-I.02) & 0.002 & SCGBID2 I.0I (95\% Cl I.00-I.0I) & 0.006 & SCGBID2 I.0I (95\% Cl I.00-1.02) & 0.005 \\
\hline & & & CAI9-9 I.00 (95\% Cl 0.99-I.0I) & 0.308 & CAI9-9 I.00 (95\% Cl 0.99-I.0I) & 0.460 \\
\hline CAI9-9 & I.0I (95\% Cl I.00-I.0I) & 0.033 & - & - & I.0I (95\% Cl I.00-I.0I) & 0.058 \\
\hline
\end{tabular}

Notes: Model I: Odds ratio adjusted for CAI9-9. Model 2: Odds ratio adjusted for CAI9-9, age and gender.

Abbreviations: AUC, area under the curve; CAI9-9, cancer antigen 19-9; Cl, confidence interval; OR, odds ratio; PODXL, podocalyxin; SCGBID2, secretoglobin family ID, member 2.

A
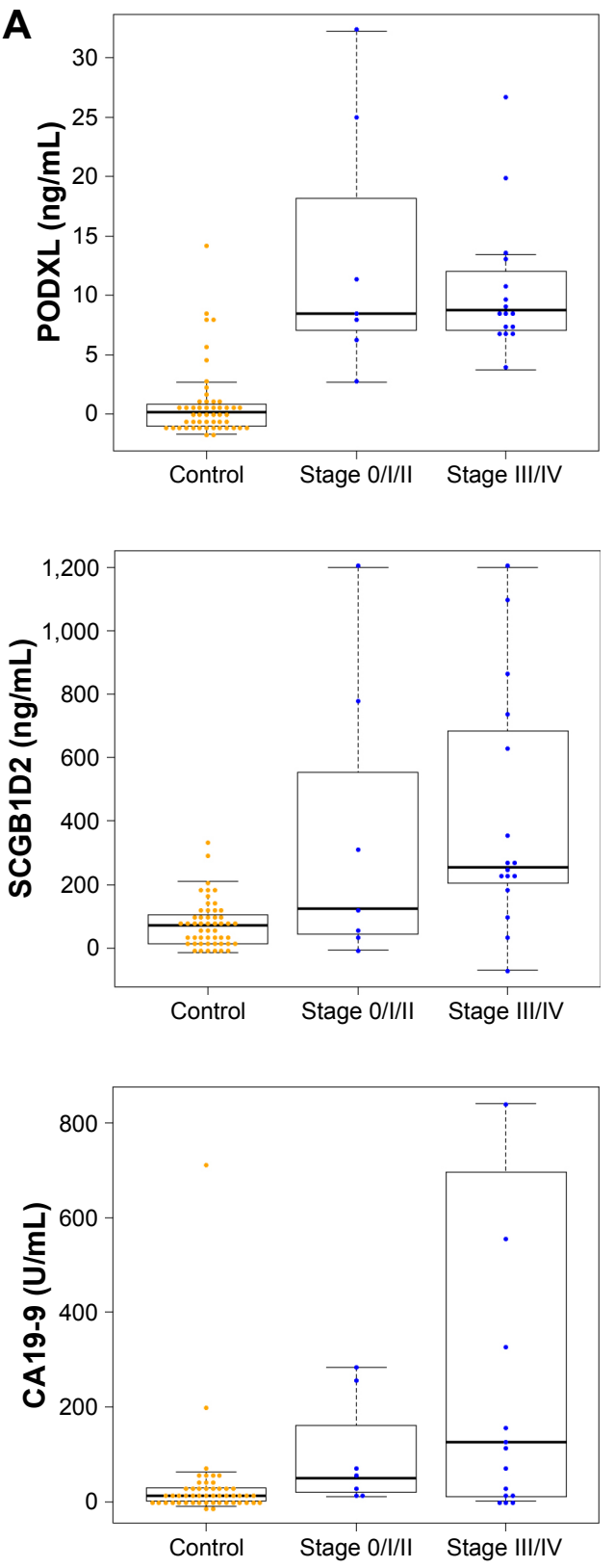
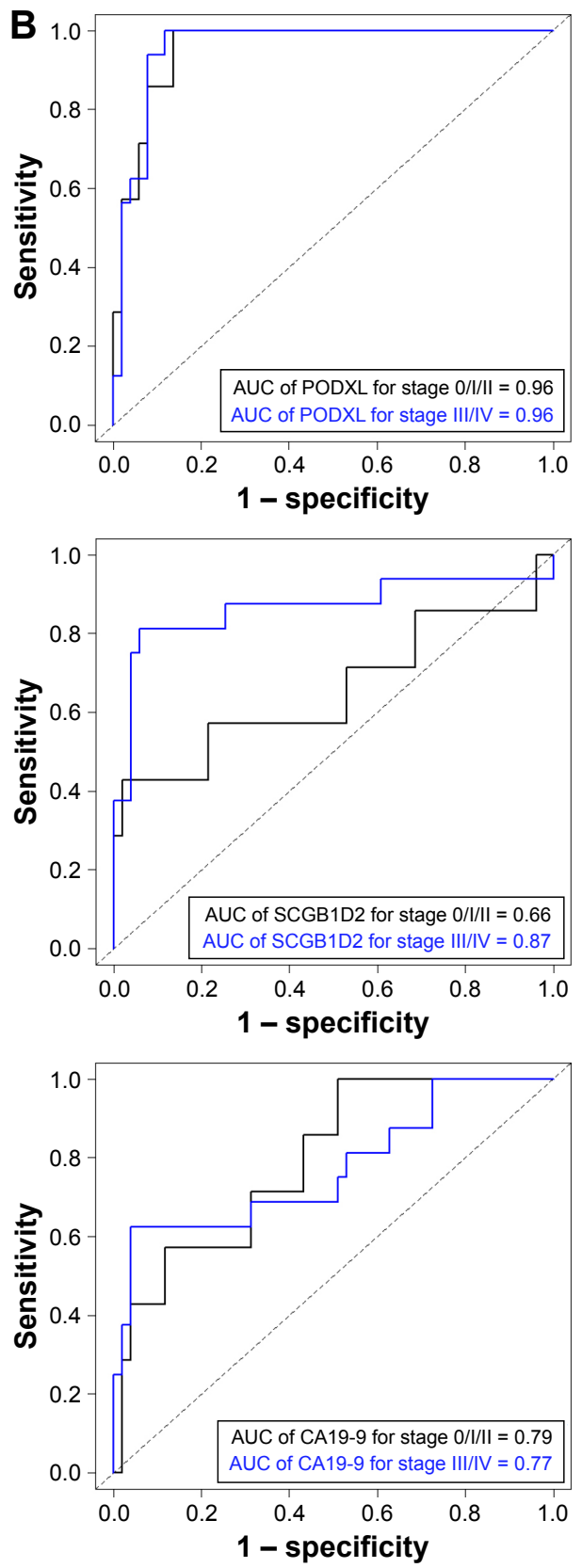

Figure 3 Determination of PODXL, SCGBID2, and CAI9-9 by ELISA in Stage 0/I/II and Stage III/IV PDACs.

Notes: (A) PODXL, SCGBID2, and CA19-9 levels in sera from Stage 0/I/II PDACs, Stage III/IV PDACs, and control individuals. The horizontal line in the middle of each box indicates the median, whereas the top and bottom borders of the box mark the 75th and 25th percentiles, respectively. The upper whisker is the 75th percentile + $(\mathrm{I} .5 \times \mathrm{IQR})$. The lower whisker is the 25th percentile - (I.5 $\times$ IQR). (B) ROC curves for the performance of PODXL, SCGBID2, and CAI9-9 from Stage 0/I/II PDACs, Stage III/IV PDACs, and control individuals.

Abbreviations: CAI9-9, cancer antigen 19-9; ELISA, enzyme-linked immunosorbent assay; IQR, interquartile range; PDAC, pancreatic ductal adenocarcinoma; PODXL, podocalyxin; ROC, receiver operating characteristic; SCGBID2, secretoglobin family ID, member 2. 
Table 7 Concentration and AUC of PODXL, SCGBID2, and CA19-9 at each clinical stage of PDAC

\begin{tabular}{|c|c|c|c|c|c|}
\hline Indicators & State & PDAC stage ${ }^{a}$ & Number & Median (IQR) & AUC $(95 \% \mathrm{Cl})$ \\
\hline \multirow[t]{3}{*}{ PODXL } & Control & & 51 & $0.15(-1.01-0.86)$ & Reference \\
\hline & PDAC & $0 / / / I I$ & 7 & $8.48(7.05-18.2)$ & $0.96(0.90-I)$ \\
\hline & & $\mathrm{III} / \mathrm{IV}$ & 16 & $8.74(7.14-11.42)$ & $0.96(0.91-1)$ \\
\hline \multirow[t]{3}{*}{ SCGBID2 } & Control & & 51 & $71.09(13.08-104.50)$ & Reference \\
\hline & PDAC & $0 / / / I I$ & 7 & I $24.50(44.52-553.00)$ & $0.66(0.37-0.95)$ \\
\hline & & $\mathrm{III} / \mathrm{IV}$ & 16 & $254.30(211.70-661.70)$ & $0.87(0.73-I)$ \\
\hline \multirow[t]{3}{*}{ CA19-9 } & Control & & 51 & $12.70(0.93-28.4 I)$ & Reference \\
\hline & PDAC & $0 / / / I I$ & 7 & $50.00(19.00-161.80)$ & $0.79(0.62-0.96)$ \\
\hline & & $\mathrm{III} / \mathrm{IV}$ & 16 & $125.40(\mid 1.02-624.20)$ & $0.77(0.62-0.92)$ \\
\hline
\end{tabular}

Note: aClassified according to the classification of the International Union against Cancer.

Abbreviations: AUC, area under the curve; CAI9-9, cancer antigen 19-9; Cl, confidence interval; IQR, interquartile range; PDAC, pancreatic ductal adenocarcinoma; PODXL, podocalyxin; SCGBID2, secretoglobin family ID, member 2.

\section{Discussion}

Kaplan-Meier plots and univariate and multivariate analyses of pathological findings have previously shown that high expressions of PODXL and SCGB1D2 are significant prognostic factors associated with worse survival after PDAC resection. ${ }^{4,5}$ Expression levels of PODXL and SCGB1D2 were evaluable in all 102 PDAC cases, and these cases were classified into low-expressing (70.6\% for PODXL and $63.7 \%$ for SCGB1D2) and high-expressing (29.4\% for PODXL and $36.3 \%$ for SCGB1D2) groups. ${ }^{4,5}$ Therefore, an increase in PODXL and SCGB1D2 within tumors would be expected to affect signaling pathways relevant to cell proliferation, invasiveness, and metastasis in PDAC. Since sensitive and specific biomarkers to identify patients with PDAC at an early stage are needed, it is important to investigate whether serum PODXL and SCGB1D2 are useful in the diagnosis of PDAC, as was done in the present study.

We previously reported that intracellular PODXL promotes PDAC cell motility and invasion by physically binding to the cytoskeletal protein gelsolin, and PODXL is a significant prognostic factor that predicts the overall survival of patients with PDAC. ${ }^{4}$ Since membranous PODXL is rarely expressed in migrating PDAC cells, intracellular PODXL bound to gelsolin may be more important for the promotion of cell motility and invasion than membranous PODXL. ${ }^{4}$ The main function of PODXL is to regulate cell morphology and adhesion by mediating the connection between intracellular proteins and extracellular ligands. ${ }^{25}$ The present study demonstrated that PODXL accumulates in the protrusions of PDAC cells and localizes to the cell surface. In addition, it was shown that PODXL is present in the media of PDAC cell cultures; however, the molecular differences (both in terms of amino acid sequence and post-translational modifications) between membrane-bound PODXL and extracellular PODXL, as well as the functions of extracellular PODXL, are currently unknown. Future studies should thus evaluate the structure of the soluble form of PODXL that can be detected in culture-conditioned media from PDAC cells.

Tumor-specific transmembrane or secretory proteins have significant advantages as biomarkers because they are present on the cell surface, within the extracellular space, or in the blood and are thus easily accessible. ${ }^{26}$ Like PODXL, SCGB1D2 was also found to accumulate in the protrusions of migrating PDAC cells, and it was detected both on the cell surface and in the culture media of PDAC cells. Western blotting using anti-PODXL antibody that recognized the extracellular domain of PODXL showed two bands of PODXL (30 kDa and $45 \mathrm{kDa}$ ) in the cell lysate of PDAC cells and a single band of PODXL (35 kDa) in the culture media of PDAC cells (Figure 1D). PODXL contains a potential $\mathrm{NH}_{2}$-terminal signal peptide, a single $\mathrm{COOH}$-terminal membrane-spanning region, and the extracellular domain containing multiple potential sites for $\mathrm{N}$ - and $\mathrm{O}$-linked glycosylation. ${ }^{23}$ It is possible that $30 \mathrm{kDa}$ and $40 \mathrm{kDa}$ PODXLs were present due to post-translational modifications, most likely glycosylation, in PDAC cells. The molecular weight of a portion of the intracellular domain of PODXL is $\sim 10 \mathrm{kDa},{ }^{23}$ and, thus, a portion of the extracellular domain of PODXL could be detected in the culture media of PDAC cells. There were also high levels of PODXL and SCGB1D2 in serum samples from PDAC patients. It is possible that the extracellular domain of PODXL was present in the serum obtained from PDAC patients. Serum SCGB1D2 was correlated with gender and serum triglyceride levels, but other clinicopathological factors were not significantly correlated with SCGB1D2 serum levels. The reasons for the observed associations between serum SCGB1D2, gender, and serum triglyceride levels are unknown; however, this should be further examined in future clinical studies.

To validate the feasibility of using serum levels of PODXL and SCGB1D2 as diagnostic tools, the sensitivity and 
specificity of these markers were compared with those of the current standard serum marker, CA19-9. PODXL performed better than serum CA19-9 when using AUC to discriminate between PDAC and control individuals; however, the increase in AUC was not significant for SCGB1D2. It was also found that the combination of SCGB1D2 and CA19-9 did not significantly increase the AUC $(0.83 ; 95 \%$ CI $0.70-0.96)$ compared with either SCGB1D2 $(0.80 ; 95 \%$ CI $0.67-0.94)$ or CA19-9 alone (0.78; 95\% CI 0.66-0.90). Importantly, these results suggest that increased expression of serum PODXL is more accurate for the diagnosis of PDAC than serum CA19-9. However, the combination of SCGB1D2 and CA19-9 does not appear to be particularly useful as a serum biomarker.

A multicenter trial comparing resection and chemoradiotherapy for locally invasive resectable PDAC (Stages IIA and IIB) showed significantly better outcomes with surgery, despite the premature termination of the trial. ${ }^{27,28}$ The circumferential encasement of the celiac axis, the hepatic artery, or the superior mesenteric artery with suspected arterial tumor infiltration (T4 or Stage III) is categorized as non-resectable in most cases. ${ }^{28,29}$ Not only could better markers improve the early diagnosis of PDAC and allow more patients to undergo curative surgical resection but they could also potentially be used for patients at high risk of developing pancreatic cancer, to identify precancerous lesions while they are amenable to cure. ${ }^{30}$ The present study included seven cases of Stage 0/I/II PDAC, and the AUC value for PODXL in the detection of these tumors was significantly higher than that for CA19-9 $(P=0.048)$. This study included only one patient in Stage 0-IB; therefore, additional validation with a larger set of serum samples covering various clinical stages, especially Stage 0-IB, is necessary to validate that PODXL is a potential biomarker for the screening of early-stage PDAC.

\section{Conclusion}

The use of serum PODXL as a biomarker achieved a sensitivity of $94.6 \%$ and a specificity of $84.3 \%$ for the detection of PDAC, at a cutoff value of $2.68 \mathrm{U} / \mathrm{mL}$. All 23 patients with PDAC (100\%) and all seven patients with Stage 0/I/II PDAC (100\%) were correctly diagnosed as positive, whereas $13.7 \%$ of the 51 control individuals were falsely diagnosed as positive. Although it was quantitatively demonstrated that the detection of PODXL in serum may provide a novel, non-invasive approach for distinguishing PDAC from control participants, the present findings are preliminary until more elaborate studies clarify that PODXL is superior to CA19-9. Further studies are required to elucidate whether PODXL is useful as a diagnostic marker for the detection of
Stage 0/I/II PDACs, as well as how much additional benefit it may add in comparison to the use of serum CA19-9. To this end, we have started a prospective clinical validation study (UMIN000021938) in the Department of Gastroenterology and Hepatology at Kochi Medical School Hospital to assess the accuracy of increased expression of PODXL in the diagnosis of PDAC and the benefit of PODXL as a potential diagnostic marker for the early detection of PDACs in comparison to CA19-9. This prospective clinical study also includes patients with chronic pancreatitis and a noncancerous pancreatic lesion, such as intraductal papillary mucinous neoplasm, to assess the utility of serum PODXL as a diagnostic marker for differentiating PDAC from other pancreatic disorders.

\section{Acknowledgments}

The authors would like to thank Miki Nishigawa and Rieko Takahashi for their excellent technical assistance. This study was supported by a Grant-in-Aid for Scientific Research (KAKENHI; 15K14396).

\section{Author contributions}

All authors contributed toward data analysis, drafting and critically revising the paper and agree to be accountable for all aspects of the work.

\section{Disclosure}

The authors report no conflicts of interest in this work.

\section{References}

1. Siegel R, Naishadham D, Jemal A. Cancer statistics, 2013. CA Cancer J Clin. 2013;63(1):11-30.

2. Hidalgo M. Pancreatic cancer. $N$ Engl J Med. 2010;362(17): $1605-1617$.

3. Locker GY, Hamilton S, Harris J, et al. ASCO 2006 update of recommendations for the use of tumor markers in gastrointestinal cancer. J Clin Oncol. 2006;24(33):5313-5327.

4. Taniuchi K, Furihata M, Naganuma S, Dabanaka K, Hanazaki K, Saibara T. Podocalyxin-like protein, linked to poor prognosis of pancreatic cancers, promotes cell invasion by binding to gelsolin. Cancer Sci. 2016; 107(10):1430-1442.

5. Taniuchi K, Furihata M, Naganuma S, et al. Elevated expression of SCGB1D2 predicts unfavorable prognosis in patients with pancreatic ductal adenocarcinoma. J Pancreas. 2017;18:216.

6. Nielsen JS, McNagny KM. The role of podocalyxin in health and disease. J Am Soc Nephrol. 2009;20(8):1669-1676.

7. Saukkonen K, Hagström J, Mustonen H, et al. Podocalyxin is a marker of poor prognosis in pancreatic ductal adenocarcinoma. PLoS One. 2015;10(6): $\mathrm{e} 0129012$.

8. Klug J, Beier HM, Bernard A, et al. Uterglobin/Clara cell $10 \mathrm{kDa}$ family of proteins: nomenclature committee report. Ann NY Acad Sci. 2000;923:348-354.

9. Ni J, Kalff-Suske M, Gentz R, Schageman J, Beato M, Klug J. All human genes of the uteroglobin family are localized on chromosome 11q12.2 and form a dense cluster. Ann N Y Acad Sci. 2000;923:25-42. 
10. Dillon DC, Reynolds LD, Retter MW, et al. Serum antibodies to lipophilin B detected in late stage breast cancer patients. Clin Cancer Res. 2003;9(2):749-754.

11. O'Brien N, Maguire TM, O'Donovan N, et al. Mammaglobin a: a promising marker for breast cancer. Clin Chem. 2002;48(8):1362-1364.

12. Goonnetilleke KS, Siriwardena AK. Systematic review of carbohydrate antigen (CA19-9) as a biochemical marker in the diagnosis of pancreatic cancer. Eur J Surg Oncol. 2007;33(3):266-270.

13. Duffy MJ, Sturgeon C, Lamerz R, et al. Tumor markers in pancreatic cancer: a European Group on Tumor Markers (EGTM) status report. Ann Oncol. 2010;21(3):441-447.

14. Brand RE, Nolen BM, Zeh HJ, et al. Serum biomarker panels for the detection of pancreatic cancer. Clin Cancer Res. 2011;17(4):805-816.

15. Iwamura T, Katsuki T, Ide K. Establishment and characterization of a human pancreatic cancer cell line (SUIT-2) producing carcinoembryonic antigen and carbohydrate antigen 19-9. Jpn J Cancer Res. 1987;78(1): 54-62.

16. Lee KM, Yasuda H, Hollingsworth MA, Ouellette MM. Notch 2-positive progenitors with the intrinsic ability to give rise to pancreatic ductal cells. Lab Invest. 2005;85(8):1003-1012.

17. Taniuchi K, Nishimori I, Hollingsworth MA. Intracellular CD24 inhibits cell invasion by posttranscriptional regulation of BART through interaction with G3BP. Cancer Res. 2011;71(3):895-905.

18. Sobin LH, Gospodarowicz MK, Witteknd C. TNM Classification of Malignant Tumors. 7th ed. New York: Wiley-Blackwell; 2009: 132-135.

19. Japan Pancreatic Society. Classification of Pancreatic Carcinoma. 2nd ed. Tokyo: Kanehara \& Co; 2003.

20. DeLong ER, DeLong DM, Clarke-Pearson DL. Comparing the areas under two or more correlated receiver operating characteristic curves: a nonparametric approach. Biometrics. 1988;44(3):837-845.
21. Youden W. Index for rating diagnostic tests. Cancer. 1950;3(1) 32-35.

22. Soureshjani MH, Kimiagari AM. Calculating the best cut off point using logistic regression and neural network on credit scoring problem - a case study of a commercial bank. Afr J Bus Manag. 2013;7:1414-1421.

23. Kershaw DB, Beck SG, Wharram BL, et al. Molecular cloning and characterization of human podocalyxin-like protein: orthologous relationship to rabbit PCLP1 and rat podocalyxin. J Biol Chem. 1997; 272(25):15708-15714.

24. Yamaji R, Chatani E, Harada N, Sugimoto K, Inui H, Nakano Y. Glyceraldehyde-3-phosphate dehydrogenase in the extracellular space inhibits cell spreading. Biochim Biophys Acta. 2005;1726(3): 261-271.

25. Thomas SN, Schnaar RL, Konstantopoulos K. Podocalyxin-like protein is an E-/L-selectin ligand on colon carcinoma cells: comparative biochemical properties of selectin ligands in host and tumor cells. Am J Physiol Cell Physiol. 2009;296(3):C505-C513.

26. Ishikawa N, Daigo Y, Yasui W, et al. ADAM8 as a novel serological and histochemical marker for lung cancer. Clin Cancer Res. 2004;10(24):8363-8370.

27. Imamura M, Doi R, Imaizumi $T$, et al. A randomized multicenter trial comparing resection and radiochemotherapy for resectable locally invasive pancreatic cancer. Surgery. 2004;136(5):1003-1011.

28. Hartwig W, Werner J, Jäger D, Debus J, Büchler MW. Improvement of surgical results for pancreatic cancer. Lancet Oncol. 2013;14(11): e476-e485.

29. Callery MP, Chang KJ, Fishman EK, et al. Pretreatment assessment of resectable and borderline resectable pancreatic cancer: expert consensus statement. Ann Surg Oncol. 2009;16(7):1727-1733.

30. Goggins M. Molecular markers of early pancreatic cancer. J Clin Oncol. 2005;23(20):4524-4531.
OncoTargets and Therapy

\section{Publish your work in this journal}

OncoTargets and Therapy is an international, peer-reviewed, open access journal focusing on the pathological basis of all cancers, potential targets for therapy and treatment protocols employed to improve the management of cancer patients. The journal also focuses on the impact of management programs and new therapeutic agents and protocols on

\section{Dovepress}

patient perspectives such as quality of life, adherence and satisfaction. The manuscript management system is completely online and includes a very quick and fair peer-review system, which is all easy to use. Visit http://www.dovepress.com/testimonials.php to read real quotes from published authors. 